\title{
A INTENSIDADE DAS AULAS DE EDUCAÇÃO FÍSICA PARA A PROMOÇÃO DA SAÚDE
}

Guilherme Rafael de Aurélio, José Ricardo Silva

Universidade Estadual Paulista - UNESP, Faculdade de Ciências e Tecnologia, Departamento de Educação Física, Presidente Prudente, SP. E-mail: guiibz @hotmail.com.

\section{RESUMO}

A atividade física é importante para a prevenção e diminuição de fatores de risco como a morbidade, obesidade e sedentarismo, e atua na melhoria da qualidade de vida. A Educação Física, historicamente, aborda a saúde em âmbito escolar. Sua especificidade permite que o professor tematize questões como fatores de risco para doenças cardiovasculares, por exemplo, cigarro, bebida alcoólica, obesidade, sedentarismo e má alimentação. Assim, este artigo objetiva demonstrar que são necessárias alterações no planejamento das aulas de Educação Física em âmbito escolar para que promova a saúde dos alunos. Na busca por este objetivo, realizamos uma pesquisa bibliográfica. Os resultados apontam que, as aulas de Educação Física não contribuem para a saúde dos praticantes. Faz-se necessário, então, uma mudança na forma de elaboração das aulas para atingir níveis de intensidade consideráveis, para promover alterações fisiológicas nos indivíduos, levando em conta fatores como gênero, biótipo e estatura.

Palavras chave: Educação Física, escola, aula, saúde, qualidade de vida.

\section{THE INTENSITY OF PHYSICAL EDUCATION CLASSES FOR HEALTH PROMOTION}

\begin{abstract}
Physical activity is important for the prevention and reduction of risk factors such as morbidity, obesity and sedentary lifestyle, and works to improve the quality of life. Physical education, historically, addresses health in the school environment. Its specificity allows the teacher discuss issues as risk factors for cardiovascular disease, such as smoking, alcohol, obesity, sedentary lifestyle and poor diet. Thus, this article aims to demonstrate that changes are needed in the planning of physical education classes in school environment to promote the health of students. In pursuit of this goal, we conducted a literature search. The results show that the physical education classes do not contribute to the health of practitioners. It is necessary, then a change in the preparation of lessons to achieve considerable levels of intensity to promote physiological changes in individuals, taking into account factors such as gender, biotype and stature.
\end{abstract}

Key words: Physical Education, school, class, health, quality of life. 


\section{INTRODUÇÃO}

Esquematicamente o corpo humano é composto por órgãos e, em conjunto formam sistemas, que inter-relacionados constituem o mecanismo biológico cada qual com suas diferenças e suas limitações. Se os corpos dos alunos forem vistos apenas como conjuntos biológicos haverá uma tendência de encará-los como parte da natureza, portanto possuiriam o mesmo corpo e a mesma capacidade. Negligenciando tais diferenças uma determinada atividade física beneficiaria apenas um determinado grupo enquanto não traria resultado benéfico para a saúde do outro (DAOLIO, 1995).

Neste trabalho, entendemos a saúde não apenas como ausência de doenças, mas também como um estado onde se encontram exaltados os valores de bem estar físico, mental e social que influenciam no comportamento humano. A saúde também é uma condição humana caracterizadas por pólos positivos e negativos. A positiva está associada à capacidade de apreciar a vida e enfrentar desafios, e a negativa estaria associada com a mortalidade e incidência de doenças (PITANGA, 2002).

Além dos valores de bem-estar, a Organização Mundial de Saúde (OMS) complementa esta concepção apontando que uma boa saúde está relacionada a qualidade de vida, ingestão balanceada de alimentos saudáveis e prática frequente de exercícios. Esses três fatores estão diretamente ligados ao equilíbrio da saúde do indivíduo. Além do mais,

[...] a atividade física relacionada à saúde aparece como um dos fatores que poderia modificar o risco dos indivíduos para adoecerem. Em primeiro lugar, existem evidências bastante significativas da influência da atividade física na melhoria da eficiência do sistema imunológico [...]. Por outro lado, a adoção de estilo de vida ativo fisicamente, irá proporcionar mudança de comportamento dos indivíduos." (PITANGA, 2002, p.51)

Contudo, o indivíduo que não pratica atividade física acaba por não procurar maneiras de se colocar em movimento, dessa forma se tornando uma pessoa fora do seu equilíbrio saudável. Esse indivíduo por não se movimentar está sujeito a aumento dos fatores de riscos de doenças ligadas aos sistemas cardiovasculares e também quadros de agravos respiratórios, além de apresentar problemas de ordem psicológica não satisfazendo seus desejos emocionais ante as situações cotidianas. O sedentarismo aparece nessa situação como principal ferramenta de lazer por não oferecer elevado gasto calórico e também pelo alto grau de prazer proporcionado pelo comodismo em que se dá. Por culpa de uma sociedade urbanizada e violenta os indivíduos ficam confinados dentro de suas casas onde tem como única opção de lazer atividades sedentárias. (BRACCO, et al, 2003)

Por outro lado, o individuo que é colocado em um estado de excesso de atividade física intensa está sujeito a, se criança, um dano no desenvolvimento cognitivo, afetivo e motor além de lesões musculoesqueléticos. As lesões que habitualmente podem vir a acontecer resultam de atividades intensas e repetitivas praticadas por indivíduos que participam de treinamentos e competições de alto nível esportivo. Quando a prática da atividade física ou treinamento se dá de maneira inadequada podem ocorrer, além das lesões, estresse, distúrbios alimentares e psicológicos. (BRACCO, et al, 2003)

Tomando as assertivas citadas acima referências, o objetivo deste artigo é demonstrar que são necessárias alterações na forma como são planejadas as aulas de educação física no âmbito escolar, para que a mesma assuma seu papel prioritário, promoção da saúde, para todos os gêneros, biótipos e estatura, ou seja, sem perder o caráter inclusivo, bem como as aulas de Educação Física escolar, da maneira que veem sendo ministradas, não promovem mudanças significativamente positivas para a saúde. Ao final deste artigo, baseado em estudos científicos, é possível constatar como alterações no planejamento das aulas de educação física podem 
proporcionar mudanças na saúde, contribuindo para o desenvolvimento de hábitos saudáveis que perduram após o período e escolarização.

\section{METODOLOGIA}

O presente artigo trata-se de uma pesquisa bibliográfica de caráter qualitativo, onde o pesquisador é o principal instrumento, os dados coletados são predominantemente descritivos e o processo é mais importante que o produto segundo Lüdke (1986). A pesquisa bibliográfica, segundo Macedo (1994), tende a ser de forma restrita, a busca por informações bibliográficas, seleção de documentos que se relacionam diretamente com o problema da pesquisa e o respectivo fichamento das referências. Desta forma, apresenta-se como primordial técnica para a elaboração desse presente trabalho a leitura e identificação das informações contidas nos materiais selecionados. Para tanto, elegemos como fontes de pesquisas periódicos especializados onde, com auxílio de conectores, por exemplo, educação física, saúde, educação física escolar e saúde, nos deparamos com algumas obras que consideramos ser pertinentes à temática abordada.

\section{RESULTADOS}

O programa de educação física apresentado aos alunos de modo geral não leva em consideração análise corporal tão pouco o nível de esforço a que esses alunos atingem durante as aulas. Desse modo, a forma como são ministradas as aulas se mostra ineficiente na valorização da melhoria da saúde e também não promove o impacto necessário para que ocorra mudança de habito nos alunos. (GUEDES, GUEDES, 2001)

Dados apresentados evidenciam que, embora as aulas de educação física atinjam $88,4 \%$ dos alunos matriculados regularmente ela não está associada a um IMC satisfatório, seja talvez pelo fato de $23 \%$ desses alunos praticarem aula uma vez na semana, $42 \%$ duas vezes na semana e apenas $23,4 \%$ tem acesso a pratica das aulas três vezes por semana. Para muitos desses alunos as aulas de educação física se caracterizam como a única oportunidade da prática de alguma atividade física. (MONEGO, JARDIM, 2006)

Em um estudo transversal realizado com o auxílio do SOFIT para se obter os níveis de atividade das aulas de educação física notou-se que durante as aulas as meninas permaneceram mais tempo desprendidas das atividades $(25,7 \%)$ e os meninos permaneceram mais tempo em pé $(47,1 \%)$, caminhando $(29,5 \%)$ e a menor parcela do tempo sendo muito ativos (10,0\%). Segundo Hino, et al (2007, p.25) a organização das aulas de Educação Física, através do SOFIT, se deu da seguinte forma: "9,5\% das aulas foram atribuídas para o gerenciamento, 2,0\% para atividades voltadas ao desenvolvimento das aptidões físicas, 53,3\% destinadas para o desenvolvimento de jogos estruturados e 35,2\% para jogos livres. "

Embora a educação física possa ser considerada disciplina de escolarização responsável pela formação dos jovens voltada ao desenvolvimento de atitudes positivas quanto à aquisição de hábitos saudáveis de prática de atividade física, pouco se conhece sobre as características dos programas oferecidos nas escolas brasileiras. [...] A qualidade e a eficiência dos programas de educação física escolar estão estreitamente associadas ao tipo de atividade que os escolares são efetivamente envolvidos durante as aulas e a proporção do tempo de participação em esforços físicos que possam induzir a adaptações fisiológicas favoráveis ao melhor funcionamento orgânico dos jovens. (GUEDES, GUEDES, p.34, 2001) 


\section{DISCUSSÃO}

O exercício físico tem papel fundamental para uma mudança de hábitos, tanto para indivíduos obesos quanto para indivíduos que procuram uma melhora na qualidade de vida e na saúde. Seu papel no combate a obesidade é importantíssimo, pois os níveis de um e outro são inversamente proporcional. (FERNANDEZ, et al, 2004). Considera-se conceito de obesidade como um acúmulo de tecido adiposo, gordura, por todo o corpo, ocasionado por doenças endócrinometabólica ou genética ou ainda por alterações na ingestão nutricional, o consumo exagerado de alimentos hipercalóricos aliado a alta ingestão de calorias diariamente tendem a um estado de obesidade e sobre peso.

A obesidade pode ser considerada tanto causa quanto conseqüência de baixos níveis de atividades [...] e a única estratégia, que em longo prazo pode ser bem-sucedida envolve a combinação do aumento da pratica de atividades, educação nutricional e modificações de comportamento. (BRACCO, et al, 2003, p.91)

Desta forma, consideramos ainda que a obesidade e o sobre peso, aliado aos chamados fatores ambientais que são hábitos não saudáveis como, cigarro e bebida alcoólica estão diretamente ligados a um aumento no fator de risco para o aparecimento de doenças. (MONEGO, JARDIM, 2006)

Uma mudança alimentar leve, reeducação alimentar e orientação nutricional com ênfase em redução de ingestão calórica aliado com atividades físicas dentro das aulas da educação física escolar já são suficientes para mostrar uma mudança corporal leve. (FERNANDEZ, et al, 2004)

A idade escolar, que compreende o período dos quatro aos dezessete anos é caracterizada como o melhor momento para o desenvolvimento e aprendizagem de hábitos saudáveis e estilo de vida ativo fisicamente, que pode ser mantido após esse período, na vida adulta. Além dos benefícios à saúde, a atividade física nos anos iniciais do período escolar também influencia na melhora do desempenho acadêmico (BRACCO, et al, 2003).

As atividades físicas durante a idade escolar apontam benefícios para a saúde musculoesquelética, redução da massa adiposa em jovem com sobrepeso, diminuição da ansiedade e de sintomas de depressão, portanto é recomendado que toda criança e adolescente sejam fisicamente ativo durante 60 minutos, na maioria dos dias da semana, para que a atividade reflita positivamente (HINO, et al, 2007).

Ao avaliar o nível de intensidade à que os estudantes estão sendo expostos é necessário ter como base para essa, à utilização da variação da freqüência cardíaca como referencial de controle dos esforços físicos. Adotando-se a freqüência cardíaca como indicador de nível de atividade física é preciso abranger uma série de outros cuidados em relação aos fatores que entornam os alunos, como, o nível de estresse onde se encontram os alunos, a carga emocional que os rodeia, a disposição desses alunos para tal atividade, tempo de exposição direta ao exercício e o tempo desprendido para atividades não relacionadas as aulas (GUEDES, GUEDES, 2001).

Diretamente relacionada a avaliação, existe uma ferramenta chamada SOFIT, criada especificamente para qualificar os índices de eficiência das aulas de educação física. Essa ferramenta foi criada para avaliar o nível de atividade, o comportamento do professor e contexto das aulas, ela utiliza um sistema composto por três fases de tomada de decisão que examina o quão ativo esta o aluno, como o tempo da aula está organizado e o comportamento do professor diante as aulas (HINO, et al, 2007).

Ainda que todos os indicadores apontem para o papel exercido pela escola no desenvolvimento motor individual, outros indicadores apontam sua ineficiência na questão dos programas sistematizados oferecidos nas aulas de educação física. Não se tem uma base de trabalho e muito pouco se conhece sobre os métodos de abordagem a que os alunos são 
expostos. As evidencias apontadas, como grande incidência de sobrepeso e obesidade coloca a prova e derrubam os conceitos de efetividade desses programas. Enfaticamente a elaboração das aulas de educação física passa por um processo muito delicado, onde se faz necessário um cuidado maior para que as aulas não caiam em monotonia perante as atividades impostas, além de ser necessário levar em conta as individualidades de cada aluno e pensar de modo que as aulas sejam inclusivas para todos, não apenas para uma pequena parcela dos alunos que tem de alguma forma uma intimidade maior com determinado tipo de atividade. É preciso também um cuidado quanto ao tempo de exposição dos alunos nas atividades para que as aulas de educação física sejam eficientes em promover uma mudança de hábitos.

Fica a critério e responsabilidade do professor de Educação Física a escolha das atividades que será passado aos alunos, porem na mesma amplitude é responsabilidade do mesmo o estudo de base para saber a quantidade de alunos de cada sexo, a partir desse dado e da série de escolarização, existem algumas atividades que proporcionam maior aumento da freqüência cardíaca que outras, o tempo que será dedicado a pratica de determinada atividade física pode interferir nas oportunidades de tornar os jovens mais ativos fisicamente (GUEDES, GUEDES, 2001).

Embora possa apresentar limitações por se tratar de uma amostragem regional, as evidências acumuladas mediante o desenvolvimento do presente estudo sugerem que os escolares envolvidos com os atuais programas de educação física escolar não estão sendo estimulados de maneira adequada quanto à intensidade e à duração dos esforços físicos, que possam repercutir favoravelmente a um melhor estado de saúde. Ainda, parece também não estarem sendo preparados para assumir valores e atitudes que lhes permitam adotar estilo de vida mais saudável e ativo fisicamente, durante e após o período de escolarização. Talvez, essa situação possa ser atribuída ao fato dos professores de educação física, que atuam na rede escolar, estarem pouco familiarizados com conceitos atualizados associados à atividade física e à saúde no contexto educacional (GUEDES, GUEDES, 2001, p. 42-43)

O educador físico precisa se propor a uma revisão da forma que se planeja as aulas de educação física, mantendo a idéia de que a mesma precisa atingir a todos os gêneros, sexo, estatura e biótipo, lembrando de fatores como intensidade da atividade, tempo de exposição ao exercício físico, tipo de atividade a ser trabalhada sem esquecer em momento algum da necessidade de ocasionar mudanças de hábitos nesses indivíduos, para que hábitos saudáveis sejam aprendidos e utilizados durante o restante da vida adulta proporcionando a esses alunos a oportunidade de declínio no risco de doenças cardiovasculares além de uma melhor expectativa de vida (DAOLIO, 1996).

\section{CONCLUSÕES}

As pesquisas apontam que a forma como as aulas de educação física são planejadas, muitas vezes utilizando programas de atividades sistematizados, não abrangendo as individualidades dos alunos e não atingindo níveis de intensidade adequado se tornam ineficientes, para propiciar aos alunos vivencias e experiências favoráveis na busca pela promoção da saúde, papel prioritário da educação física de âmbito escolar.

Analisando como um todo, as aulas de Educação Física ministradas nas escolas, na maioria das vezes por utilizar atividades padronizadas ligadas aos esportes elaborados (futebol, vôlei, basquete, etc.), não atingem os índices necessários de intensidade para promover alterações no estado de saúde dos alunos. Desta forma o professor deve assumir atitudes que levem em consideração atividades que não prejudique o desenvolvimento do individuo, mas proporcione 
intensidade adequada, tempo de exposição apropriado e freqüência para que ocorram alterações fisiológicas favoráveis ao estilo de vida ativo fisicamente no praticante, dessa maneira promovendo melhorias na saúde e mudanças de hábitos no aluno.

\section{REFERÊNCIAS}

DAOLIO, J., Educação física escolar: em busca da pluralidade, Rev. paul. Educ. Fís., São Paulo, supl.2, p.40-42, 1996. Disponível em:

http://citrus.uspnet.usp.br/eef/uploads/arquivo/v10\%20supl2\%20artigo7.pdf, Acessado em: $14 / 07 / 2016$

MONEGO, E. T., JARDIM, P. C. B. V., Determinantes de riscos para doenças cardiovasculares em escolares, Arquivos Brasileiros de Cardiologia - Volume 87, № 1, Julho 2006. Disponível em: http://www.scielo.br/pdf/abc/v87n1/a06v87n1.pdf, Acessado em: 10/07/2016.

GUEDES, D. P., GUEDES, J. E. R.P., Esforços físicos nos programas de educação física escolar, Rev. paul. Educ. Fís., São Paulo, 15(1):33-44, jan./jun. 2001. Disponível em:

http://citrus.uspnet.usp.br/eef/uploads/arquivo/v15\%20n1\%20artigo3.pdf, Acessado em: 14/07/2016.

LÜDKE, Menga. Pesquisa em educação: abordagens qualitativas / Menga Lüdke, Marli E. D. A. André. - São Paulo: EPU, 1986.

HINO, A. A. F., et al. Observações dos níveis de atividade física, contexto das aulas e comportamento do professor em aulas de educação física do ensino médio da rede publica, Revista Brasileira de Atividade Física \& Saúde, p.21-31, 2007. Disponível em:

https://periodicos.ufpel.edu.br/ojs2/index.php/RBAFS/article/view/818/825, Acessado em: 14/07/2016.

PITANGA, F. J. G., Epidemiologia, atividade física e saúde, Rev. Bras. Ciên. e Mov. Brasília v.10 n. 3 p. 49-54 julho 2002. Disponível em:

https://portalrevistas.ucb.br/index.php/RBCM/article/viewFile/463/489, Acessado em: 14/07/2016.

MACEDO, N. D., Iniciação à pesquisa bibliográfica: guia do estudante para a fundamentação de trabalhos de pesquisa, 2. ed revista, São Paulo: edições Loyola, 1994 